\title{
Closure of a post-pneumonectomy bronchopleural fistula with fibrin sealant (Tisseel)
}

\author{
ROD T ONOTERA, HELMUT W UNRUH \\ From the Department of Surgery, University of Manitoba, Winnipeg, Manitoba, Canada
}

\begin{abstract}
A persistent post-pneumonectomy bronchopleural fistula and empyema were successfully treated by draining and cleansing the empyema cavity and then occluding the fistula with fibrin sealant.
\end{abstract}

The mortality and morbidity associated with postpneumonectomy bronchopleural fistula complicated by empyema are considerable' ${ }^{\prime}$ and its surgical treatment may be both extensive and complicated. Surgical procedures include chronic open drainage, attempts at direct stump closure with intercostal muscle reinforcement, thoracoplasty with or without extrathoracic chest wall muscle transposition, and trans-sternal bronchial closure. Detachable balloon occlusion $^{2}$ and use of an omental flap ${ }^{3}$ have recently been proposed. An alternative approach is to close the bronchopleural fistula with a sealing compound applied directly to the fistula through the flexible bronchoscope. ${ }^{4-7}$

We report the successful management of a post-pneumonectomy bronchopleural fistula by the use of the two component fibrin sealant Tisseel preceded by open drainage of the empyema.

\section{Case report}

A 55 year old man, who two years previously had undergone a pulmonary wedge resection for metastatic colonic adenocarcinoma of the right upper lobe, developed a recurrence of the adenocarcinoma at the operative site. A complete pneumonectomy was performed. The bronchus was closed with a TA-55 surgical stapler (Autosuture Canada Ltd, Ville St Laurent, Quebec). On the sixth postoperative day the patient developed a low grade fever with a raised white blood count, and he began to expectorate sputum that was thin and blood stained, resembling serous fluid. The diagnosis of empyema secondary to bronchopleural fistula was made and confirmed by fibreoptic bronchoscopy. The fistula measured $3 \mathrm{~mm}$ and was situated at the lateral aspect of the bronchial closure. A pleurocutaneous fistula was established by resecting two adjacent $8 \mathrm{~cm}$ segments of rib to establish open drainage. ${ }^{8} \mathrm{~A}$ thoracoscope was inserted through the Eloesser flap and this confirmed the presence of a bronchopleural fistula associated with substantial infection in the postpneumonectomy space.

The pneumonectomy space was treated by twice daily

Address for reprint requests: Dr H W Unruh, Respiratory Hospital, 810 Sherbrook Street, Winnipeg, Manitoba, Canada R3A 1 R8.

Accepted 5 January 1988 packing of the cavity with saline soaked gauze. The patient's temperature and white blood count returned to normal. Two weeks later, when thoracoscopy was repeated, the fistula was still present and unchanged in size. The previous signs of acute infection and inflammation were resolving satisfactorily. One millilitre of fibrin sealant was then applied directly to the pleural side of the fistula through the thoracoscope with the Duploject $1507 \mathrm{~F} 150 \mathrm{~cm}$ four lumen catheter (Immuno AG, Vienna). The four lumen catheter is required to avoid premature mixing of the fibrin compound with its thrombin activator. The third lumen delivers compressed air to spray the fibrin sealant. The fourth lumen is for a guide wire. Topical oropharyngeal anaesthesia with lignocaine and mild intravenous sedation were used to pass the rigid $8.5 \mathrm{~mm}$ bronchoscope. A second application of fibrin sealant was made to the bronchial side of the fistula. The rigid bronchoscope was used to allow passage of the multiple lumen catheter.

The pneumonectomy cavity was again loosely packed with a long saline soaked gauze. The packing was changed twice daily. The fistula was no longer audible at the time of dressing changes. The patient was discharged on the fourth postoperative day. Twice daily packings of the cavity were continued at home for six weeks. The patient was readmitted and thoracoscopy and bronchoscopy were repeated. There were now no signs of infection in the pneumonectomy space and the fistula was closed. About $750 \mathrm{~cm}^{3}$ of $0.25 \%$ neomycin in normal saline solution was instilled into the pneumonectomy space to fill the cavity completely.' Local anaesthesia was infiltrated and the Eloesser flap closed to ensure a watertight closure. Follow up examination four months later showed no recurrence of the bronchopleural fistula and no evidence of empyema.

\section{Discussion}

Treatment of bronchopleural fistulas with endoscopically delivered compounds of various types has been described by several authors. Jessen and Sharma ${ }^{4}$ first reported the successful closure of a post-pneumonectomy bronchopleural fistula with the help of fibrin sealant. Thoracotomy with resuturing of the bronchial stump was performed but the fistula recurred. Fibrin sealant was delivered endoscopically to the bronchial side of the fistula on three separate occasions. This eventually obliterated the leak. Waclawiczek and Chmelizek ${ }^{5}$ described the successful closure of bronchopleural fistulas in pigs using only the fibrin sealant method without an additional surgical procedure. Glover et $a l^{6}$ reported two cases in which post-lobectomy bronchopleural fistulas were obliterated with fibrin sealant. Both patients were treated with closed chest tube drainage and fibrin 
sealant delivered endoscopically to the bronchial aspect of the fistula.

Our management of post-pneumonectomy bronchopleural fistula with empyema differs in that it incorporates both the Clagett two stage method ${ }^{9}$ of empyema treatment and the use of fibrin sealant. Torre $e t \mathrm{al}^{7}$ recently reported three cases and reviewed published reports on endoscopic glueing of bronchopleural fistulas. ${ }^{5}$ The review included one patient treated successfully with fibrin sealant and six with isobutyl cyanoacrylate. Generally drainage was achieved with only an intercostal drainage tube with or without irrigation. We took a more aggressive approach to drainage with the establishment of an open thoracostomy. This allowed direct inspection of the fistula and examination of the post-pneumonectomy space to ensure that all residual infection had cleared before drainage was discontinued. If occlusion of the fistula by fibrin sealant fails it can be reapplied and there will be no untoward effects as complete and adequate drainage is present. Other surgical approaches to bronchopleural fistula can still be initiated at any time. We believe that effective occlusion of a bronchopleural fistula with fibrin sealant can be accomplished by application of fibrin sealant to both sides of the fistula in the presence of a clean, uninfected environment. This is best established with the creation of an open thoracostomy as described by Eloesser. $^{8}$

\section{References}

1 De la Rocha AG. Empyema thoracis. Surg Gynecol Obstet 1982;155:839-45.

2 Courtheoux P, Letourneux M, Jehan A, Theron J. Use of a detachable 2-compartment balloon in the treatment of a $\vec{D}$ bronchopleural fistula. Ann Radiol 1985;28:560-2.

3 Fujio A, Matsui T, Asakura A, Kitano M. The use of omental flap to close the post-pneumonectomy empyema space with bronchial fistula-a case report. Nippon Kyobu Geka Gakkai Zasshi $\vec{\bigcirc}$ 1986;34:1174-7.

4 Jessen C, Sharma P. Use of fibrin glue in thoracic surgery. Ann $\vec{\omega}$ Thorac Surg 1985;39:521-4.

5 Waclawiczek HW, Chmelizek F. Endoscopic treatment of bronchus stump fistulae following pneumonectomy with fibrin sealant in domestic pigs. $J$ Thorac Cardiovasc Surg 1985;33: $\vec{\omega}$ 344-6.

6 Glover W, Chavis TV, Daniel TM, Kron IL, Spotnitz WD. Fibrin N glue application through the flexible fiberoptic bronchoscope: $\overrightarrow{0}$ closure of bronchopleural fistulas. J Thorac Cardiovasc Surg $\vec{G}$ 1987;93:470-2.

7 Torre M, Chiesa G, Ravini M, Vercelloni M, Belloni PA. 근 Endoscopic gluing of bronchopleural fistulas. Ann Thorac Surg 1987;43:295-7.

8 Eloesser L. An operation for tuberculous empyema. Surg Gynecol $\mathbb{D}$ Obstet 1935;60:1096-7.

9 Clagett OT, Geraci JE. A procedure for the management of postpneumonectomy empyema. $J$ Thorac Cardiovasc Surg 1963; Ф 45:141-5. 